\title{
COLLECTIVE EFFECTS ANALYSIS FOR THE BERKELEY FEMTOSOURCE ${ }^{*}$
}

\author{
J. Corlett, S. De Santis, A. Wolski, A. Zholents LBNL, Berkeley, CA 94720, USA
}

\begin{abstract}
We present an overview of the collective effects in a proposed ultrafast $\mathrm{x}$-ray facility, based on a recirculating linac. The facility requires a small vertical ewmittance of $0.4 \mathrm{~mm}$-mrad and is designed to operate with a "flat bunch" with a large aspect ratio of emettances. Emittance control from the electron source at the RF photocatode to the photon production chain of undulators, and understanding and the mitigation of collective effects is critical to a successful machine operation. Key aspects of accelerator physics involved in beam break-up, coherent synchrotron radiation, resistive wall impedance and other effects have been addressed and reported here.
\end{abstract}

\section{INTRODUCTION}

The proposed Berkeley Femtosecond X-Ray Facility [1] (LUX) is based on a $750 \mathrm{MeV}$ superconducting recirculated linac. It accelerates up to $3.1 \mathrm{GeV}$ a flat electron beam, which is subsequently used to generate ultra-short X-ray pulses. It is vital to preserve a small vertical emittance throughout the machine since the synchrotron light is produced after the bunch has been rotated by an RF crab cavity.

In this paper we investigate the threee main mechanisms that can lead to a degradation of machine performance by $i$ emittance or energy spread increase: wakefields in the main linac and the preinjector; resistive wall impedance in the vacuum chamber; and coherent synchrotron radiation.

We also report on the strategies we plan using to overcome these problems.

The calculations presented in this paper concern an earlier, lower energy version of the machine, using a 600 $\mathrm{MeV}$ linac. Calculations with the latest parameters are underway and the final results presented here are anyway relevant for the new design, since it is shown that the higher energy parts of the machine contribute much less to the emittance growth.

\section{WAKE FIELDS}

The performance LUX depends on preservation of low vertical emittance through the linac and the arcs. The short-range transverse wake fields from the linac cavities are a potential source of vertical emittance growth, and the effects need to be carefully evaluated. The size of the transverse kick from the wake fields increases with increasing offset of the bunch from the axis of the cavity, so there are possible implications for the alignment of the cavities and orbit control.

It is possible to arrive at a semi-analytical estimate of the effects of the wake fields of the linac, which was presented in [2]. However, a more complete investigation including the nominal bunch distribution and tracking through the arcs requires a tracking code. For the present studies, we have used MERLIN [3], which allows the simulation of all the required effects. We also present some estimates of the long-range wake fields influence on the emittance growth.

\section{Short-range wake fields}

The present design of the linac uses the TESLA 9 cell, $1.3 \mathrm{GHz}$ superconducting cavities for an analytical expression for the wake fields is reported in [4] while the lattice design used for the arc tracking can be found in [5]. For an exhaustive report of the technical details of the tracking see [6].

To verify the wake field model in MERLIN, we first tracked a bunch with nominal 2 ps bunch length, and (effectively) zero transverse emittance through four passes of the linac. In this case, the bunch was injected so as to have a fixed vertical offset in each cavity, and the bunch was taken straight from the end of the linac on each pass and re-injected at the start of the linac: i.e. we modeled the effects of perfectly achromatic arcs with integer betatron phase advances.

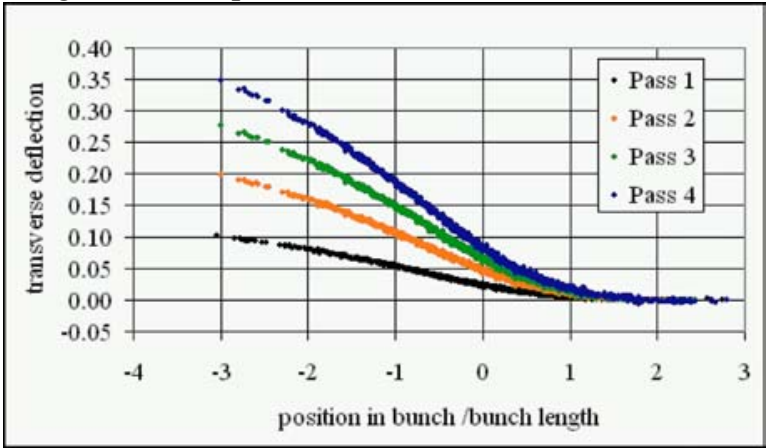

Figure 1. Transverse deflection of a 2 ps zero-emittance bunch, through four consecutive passes at constant vertical offset through the linac.

The results in Fig.1 can be compared with our analytical solution for the vertical displacement at the end of each linac pass:

$$
\begin{aligned}
y_{(1)}(z)=y_{0}+y_{0} A_{0}(z)\left[\frac{\Delta \gamma}{\gamma_{i}}\right. & \left.-\ln \left(1+\frac{\Delta \gamma}{\gamma_{i}}\right)\right]+ \\
& +y_{0}^{\prime} L \frac{\gamma_{i}}{\Delta \gamma} \ln \left(1+\frac{\Delta \gamma}{\gamma_{i}}\right)
\end{aligned}
$$

where

\footnotetext{
* Work supported by the US DoE under contract No. DE-AC03-76SF00098
} 


$$
A_{0}(z)=\frac{r_{0} \gamma_{i} L^{2}}{\Delta \gamma^{2}} \int_{z}^{\infty} \rho\left(z^{\prime}\right) W_{\perp}\left(z^{\prime}-z\right) d z^{\prime}
$$

$\gamma_{i}$ is the initial energy, $\Delta \gamma$ the energy gain, $L$ the linac length, $y_{0}$ the initial vertical offset and $y_{0}^{\prime}$ the initial vertical angle, and $z$ the position inside the electron bunch ( $z=0$ being the bunch centre).

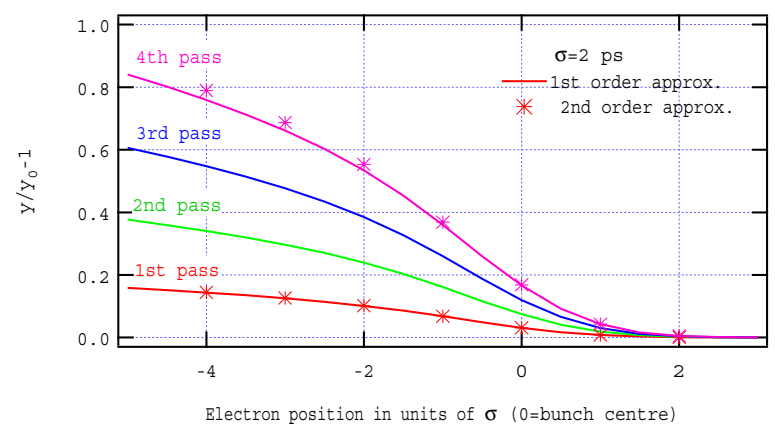

Figure 2. Cumulative transverse displacement (relative to the bunch head), as a function of the electron position within the bunch, after each linac pass.

The apparent discrepancy between Figs. 1 and 2 is entirely explained by the factor $L^{2}$ in Eq.(2): the analytical model assumes a linac completely filled with RF cavities, with no drift spaces. Once the actual filling factor of about $80 \%$ is taken into account, the two results present a very good agreement.

To estimate the emittance growth from the wake fields in a linac with vertical cavity misalignments, we tracked a bunch (of 10,000 macroparticles) with the nominal parameters as specified in Table 1 from the entrance to the linac to the exit after the fourth pass. Vertical misalignments with a range of rms values (and a cut-off at 5 sigma) were applied to the cavities; as mentioned above, the beam sees the same misalignments in the simulation on each pass. The cavity misalignments were the only imperfections applied to the machine; the arc optics and alignment were as designed. For large cavity misalignments, there is significant orbit distortion that is expected to lead to vertical emittance growth from coupling in the arc sextupoles. No distinction was made between cryostats; in reality, cavity offsets are expected to be correlated according to which cryostat they are in. Since we find that the wake field effects for any reasonable cavity misalignments are so small, our conclusions are not likely to be affected by the fact that we have neglected this effect.

Table 1: Bunch parameters used in wakefield simulations.

\begin{tabular}{|l|l|}
\hline Initial bunch energy & $120 \mathrm{MeV}$ \\
\hline Bunch charge & $1 \mathrm{nC}$ \\
\hline Bunch length & $2 \mathrm{ps}$ \\
\hline Energy spread & $10^{-3}$ \\
\hline Horizontal emittance (normalized) & $20 \mu \mathrm{m}$ \\
\hline Vertical emittance (normalized) & $0.4 \mu \mathrm{m}$ \\
\hline
\end{tabular}

Results of tracking with the sextupoles turned on are shown in Fig.3; results with the sextupoles off are shown in Fig.4. It appears that the wake fields make negligible contribution to the emittance growth. The wake field cancellation resulting from the phase advance across the arcs can easily reduce the head-tail displacement by more than an order of magnitude; in which case, we would expect to see an emittance growth of the order $5 \%$ (with 2 $\mathrm{mm}$ rms vertical cavity misalignment).

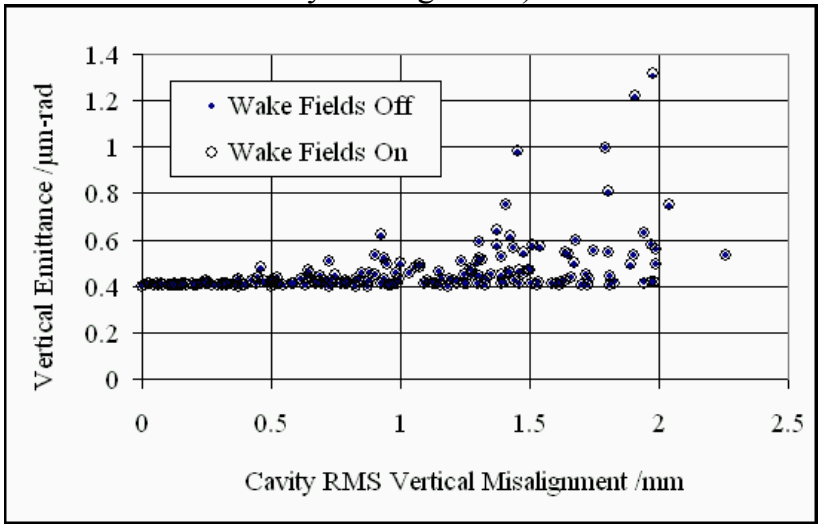

Figure 3. Results of tracking a nominal bunch from the first entrance of the linac through four passes (including the arcs) to the fourth exit from the linac. The arc sextupoles were turned on.

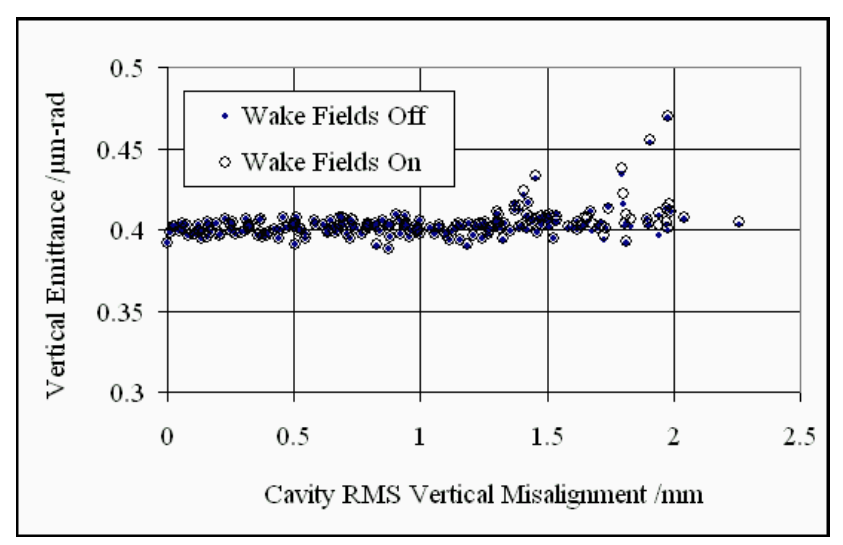

Figure 4. Results of tracking a nominal bunch from the first entrance of the linac through four passes (including the arcs) to the fourth exit from the linac. The arc sextupoles were turned off.

With the sextupoles turned on, any emittance growth from the wake fields is hidden by the very much larger emittance growth from the coupling. This should be easily fixed by proper orbit control. 


\section{Long-range wake fields}

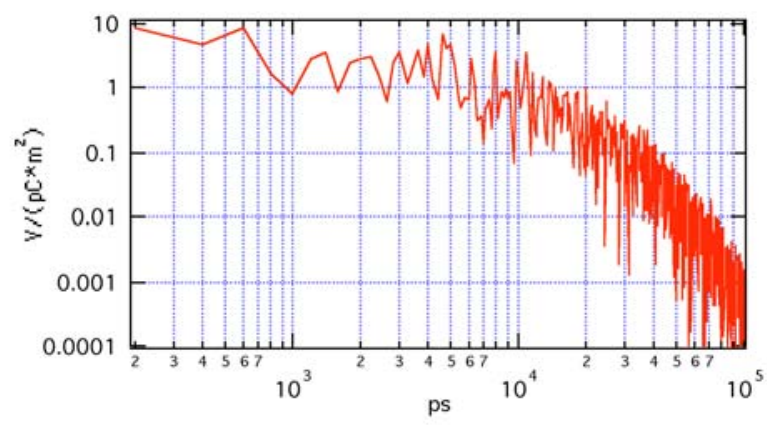

Figure 5. Long-range transverse wakefield with a $0.1 \%$ random detuning in the RF cavities HOMs.

Since a bunch in LUX is recirculated four times through the main linac, we also investigate the influence of the transverse long-range wakefield on the bunch emittance. An estimate of such wakefield can be obtained by adding up the transverse wake from all the high-order modes of the 32 linac cavities. Figure 5 shows that the field amplitude decays by only a factor of 10 in the typical recirculation time of less than $1 \mu$ s. The long-range transverse wakefield is, anyway, fairly constant over the bunch length and, therefore, its effect translates in just a small additional dislacement of the entire bunch (Fig.6), which doesn't affect the vertical emittance.

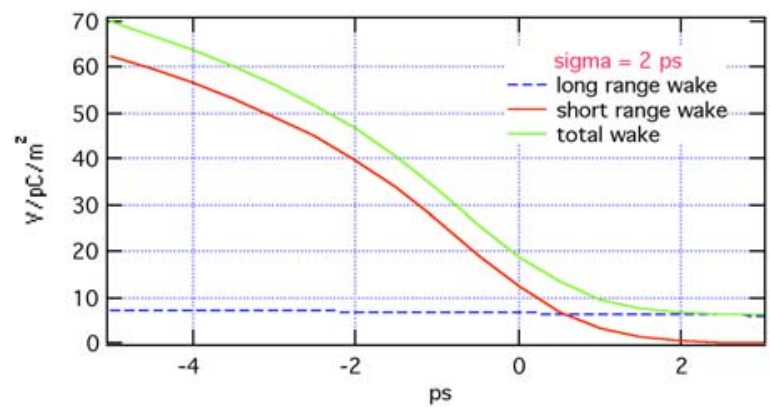

Figure 6. Total vertical displacement along a 2 ps bunch from short and long range transverse wakefields.

\section{RESISTIVE WALL IMPEDANCE}

The resistive wall impedance also induces a vertical deflection along a bunch throughout the machine, which leads to a dilution of the vertical emittance. The resistive wall effects are worse at lower energies, for shorter bunches and for narrower apertures of the vacuum chamber. Figure 7 shows the oscillation of the bunch tail along the first arc for a beam pipe radius of $4.5 \mathrm{~mm}$ and an initial vertical offset of $100 \mu \mathrm{m}$. The tracking code results agree very well with a simple analytical model.

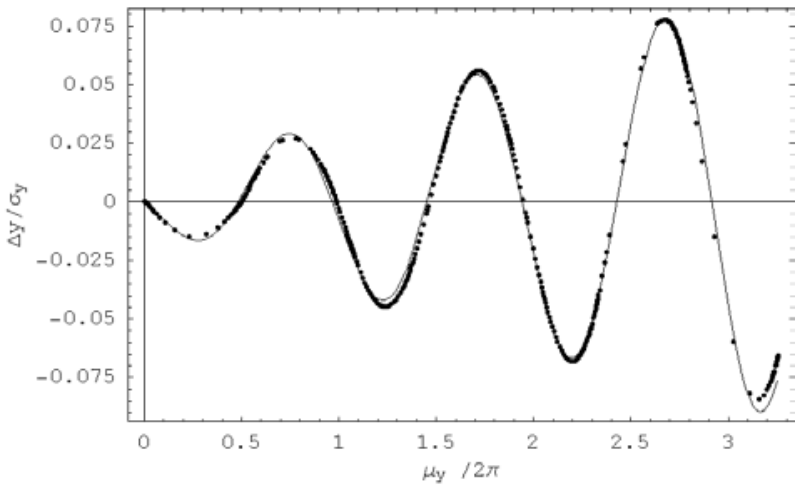

Figure 7. Vertical deflection of the bunch tail along an arc. Tracking (dots) and analytical model (solid line).

It can be seen that the resistive wall contribution is limited to less than $10 \%$ of the vertical beam size. This figure is expected to improve, as we expect to have an orbit control significantly better than $100 \mu \mathrm{m}$. The effects in the other arcs will be significantly smaller, because of the higher beam energy, so that we don't expect the resistive wall impedance to limit the performance of the light source.

\section{COHERENT SYNCHROTRON RADIATION}

Coherent syncrotron radiation (CSR) causes a variable energy loss of electrons in along a bunch which, in turn, leads to emittance increase in the orbit plane. Since we have a relatively large horizontal emittance, a small emittance growth does't seem to be harmful. Therefore, we are mainly concerned by the energy loss because of the consequent increase in the energy spread.

\section{CONCLUSIONS}

We have investigated several fundamental aspects of the beam dynamics in LUX. We are mainly concerned with the preservation throughout the machine of the vertical emittance and the energy spread, which are vital for the generation of ultra-short x-ray pulses. Our results show that, while one must always keep these aspects present in the design phase, they don't present insurmountable problems towards obtaining the machine design parameters.

\section{REFERENCES}

[1] J. Corlett, et al. "A Recirculating Linac-Based Facility for Ultrafast X-Ray Science", these proceedings.

[2] S. De Santis and A. Zholents, "Beam Break Up Analysis for the Berkeley Femtosource", EPAC 2002.

[3] N.J. Walker's MERLIN (a C++ class library for accelerator beamline simulation) can be found at http://www.desy.de/ njwalker/MerlinII/index.htm

[4] "TESLA Technical Design Report", DESY 2001011, March 2001. 
[5] A. Zholents et al, "Initial Lattice Studies for the Berkeley Femtosecond X-Ray Light Source", proceedings EPAC 2002, Paris, France, June 2002.

[6] A. Wolski, "Tracking Studies of RF Cavity Transverse Wake Fields in the Berkeley
Femtosecond Light Source", CBP Tech Note-265 (2002).

[8] J.M. Byrd, et al. "Transient Beam loading Effects in Harmonic RF Systems for light Sources", Phys. Rev. ST-AB 5, 092001, 2002. 